\title{
MULTIVARIATE FRACTIONAL REPRESENTATION FORMULA AND OSTROWSKI TYPE INEQUALITY
}

\author{
GEORGE A. ANASTASSIOU
}

\begin{abstract}
Here we derive a multivariate fractional representation formula involving ordinary partial derivatives of first order. Then we prove a related multivariate fractional Ostrowski type inequality with respect to uniform norm.
\end{abstract}

\section{INTRODUCTION}

Let $f:[a, b] \rightarrow \mathbb{R}$ be differentiable on $[a, b]$, and $f^{\prime}:[a, b] \rightarrow \mathbb{R}$ be integrable on $[a, b]$, then the following Montgomery identity holds [3]:

$$
f(x)=\frac{1}{b-a} \int_{a}^{b} f(t) d t+\int_{a}^{b} P_{1}(x, t) f^{\prime}(t) d t
$$

where $P_{1}(x, t)$ is the Peano kernel

$$
P_{1}(x, t)= \begin{cases}\frac{t-a}{b-a}, & a \leq t \leq x, \\ \frac{t-b}{b-a}, & x<t \leq b,\end{cases}
$$

The Riemann-Liouville integral operator of order $\alpha>0$ with anchor point $a \in \mathbb{R}$ is defined by

$$
\begin{gathered}
J_{a}^{\alpha} f(x):=\frac{1}{\Gamma(\alpha)} \int_{a}^{x}(x-t)^{\alpha-1} f(t) d t, \\
J_{a}^{0} f(x):=f(x), \quad x \in[a, b] .
\end{gathered}
$$

Properties of the above operator can be found in [4].

When $\alpha=1, J_{a}^{1}$ reduces to the classical integral.

In [1] we proved the following fractional representation formula of Montgomery identity type.

2010 Mathematics Subject Classification. 26A33, 26D10, 26D15.

Key words and phrases. Multivariate fractional integral, representation formula, multivariate Ostrowski inequality. 
Theorem 1. Let $f:[a, b] \rightarrow \mathbb{R}$ be differentiable on $[a, b]$, and $f^{\prime}:[a, b] \rightarrow \mathbb{R}$ be integrable on $[a, b], \alpha \geq 1, x \in[a, b)$. Then

$$
\begin{aligned}
f(x)=(b-x)^{1-\alpha} \Gamma(\alpha) & \\
& \cdot\left\{\frac{J_{a}^{\alpha} f(b)}{b-a}-J_{a}^{\alpha-1}\left(P_{1}(x, b) f(b)\right)+J_{a}^{\alpha}\left(P_{1}(x, b) f^{\prime}(b)\right)\right\} .
\end{aligned}
$$

When $\alpha=1$ the last (5) reduces to classic Montgomery identity (1).

We may rewrite (5) as follows

$$
\begin{aligned}
& f(x)=(b-x)^{1-\alpha}\left[\frac{1}{b-a} \int_{a}^{b}(b-t)^{\alpha-1} f(t) d t\right. \\
& \left.-(\alpha-1) \int_{a}^{b}(b-t)^{\alpha-2} P_{1}(x, t) f(t) d t+\int_{a}^{b}(b-t)^{\alpha-1} P_{1}(x, t) f^{\prime}(t) d t\right] .
\end{aligned}
$$

In this article based on (5), we establish a multivariate fractional representation formula for $f(x), x \in \prod_{i=1}^{m}\left[a_{i}, b_{i}\right] \subset \mathbb{R}^{m}$, and from there we derive an interesting multivariate fractional Ostrowski type inequality.

\section{MAIN RESUlts}

We make

Assumption 2. Let $f \in C^{1}\left(\prod_{i=1}^{m}\left[a_{i}, b_{i}\right]\right)$.

Assumption 3. Let $f: \prod_{i=1}^{m}\left[a_{i}, b_{i}\right] \rightarrow \mathbb{R}$ be measurable and bounded, such that there exist $\frac{\partial f}{\partial x_{j}}: \prod_{i=1}^{m}\left[a_{i}, b_{i}\right] \rightarrow \mathbb{R}$, and it is $x_{j}$-integrable for all $j=1, \ldots, m$. Furthermore $\frac{\partial f}{\partial x_{i}}\left(t_{1}, \ldots, t_{i}, x_{i+1}, \ldots, x_{m}\right)$ it is integrable on $\prod_{j=1}^{i}\left[a_{j}, b_{j}\right]$, for all $i=1, \ldots, m$, for any $\left(x_{i+1}, \ldots, x_{m}\right) \in \prod_{j=i+1}^{m}\left[a_{j}, b_{j}\right]$.

Convention 4. We set

$$
\prod_{j=1}^{0} \cdot=1 .
$$

Notation 5. Here $x=\vec{x}=\left(x_{1}, \ldots, x_{m}\right) \in \mathbb{R}^{m}, m \in \mathbb{N}-\{1\}$. Likewise $t=\vec{t}=\left(t_{1}, \ldots, t_{m}\right)$, and $d \vec{t}=d t_{1} d t_{2} \ldots d t_{m}$. We denote the kernel

$$
P_{1}\left(x_{i}, t_{i}\right)= \begin{cases}\frac{t_{i}-a_{i}}{b_{i}-a_{i}}, & a_{i} \leq t_{i} \leq x_{i}, \\ \frac{t_{i}-b_{i}}{b_{i}-a_{i}}, & x_{i}<t_{i} \leq b_{i},\end{cases}
$$

We need 
Definition 6. [see [2]] Let $\prod_{i=1}^{m}\left[a_{i}, b_{i}\right] \subset \mathbb{R}^{m}, m \in \mathbb{N}-\{1\}, a_{i}<b_{i}$, $a_{i}, b_{i} \in \mathbb{R}$. Let $\alpha>0, f \in L_{1}\left(\prod_{i=1}^{m}\left[a_{i}, b_{i}\right]\right)$. We define the left mixed Riemann-Liouville fractional multiple integral of order $\alpha$ :

$\left(I_{a+}^{\alpha} f\right)(x):=\frac{1}{(\Gamma(\alpha))^{m}} \int_{a_{1}}^{x_{1}} \ldots \int_{a_{m}}^{x_{m}}\left(\prod_{i=1}^{m}\left(x_{i}-t_{i}\right)\right)^{\alpha-1} f\left(t_{1}, \ldots, t_{m}\right) d t_{1} \ldots d t_{m}$,

where $x_{i} \in\left[a_{i}, b_{i}\right], i=1, \ldots, m$, and $x=\left(x_{1}, \ldots, x_{m}\right), a=\left(a_{1}, \ldots, a_{m}\right)$, $b=\left(b_{1}, \ldots, b_{m}\right)$.

We present the following multivariate fractional representation formula

Theorem 7. Let $f$ as in Assumption 2 or Assumption 3, $\alpha \geq 1, x_{i} \in\left[a_{i}, b_{i}\right)$, $i=1, \ldots, m$. Then

$$
\begin{aligned}
f\left(x_{1}, \ldots, x_{m}\right)=\frac{\left(\prod_{i=1}^{m}\left(b_{i}-x_{i}\right)\right)^{1-\alpha}(\Gamma(\alpha))^{m}}{\prod_{i=1}^{m}\left(b_{i}-a_{i}\right)}\left(I_{a+}^{\alpha} f\right)(b) \\
\quad+\sum_{i=1}^{m} A_{i}\left(x_{1}, \ldots, x_{m}\right)+\sum_{i=1}^{m} B_{i}\left(x_{1}, \ldots, x_{m}\right),
\end{aligned}
$$

where for $i=1, \ldots, m$ :

$$
\begin{gathered}
A_{i}\left(x_{1}, \ldots, x_{m}\right):=\frac{-(\alpha-1)\left(\prod_{j=1}^{i}\left(b_{j}-x_{j}\right)\right)^{1-\alpha}}{\prod_{j=1}^{i-1}\left(b_{j}-a_{j}\right)} \int_{\prod_{j=1}^{i}\left[a_{j}, b_{j}\right]}\left(\prod_{j=1}^{i-1}\left(b_{j}-t_{j}\right)\right)^{\alpha-1} \\
\cdot\left(b_{i}-t_{i}\right)^{\alpha-2} P_{1}\left(x_{i}, t_{i}\right) f\left(t_{1}, \ldots, t_{i}, x_{i+1}, \ldots, x_{m}\right) d t_{1} \ldots d t_{i}, \quad(11)
\end{gathered}
$$

and

$$
\begin{aligned}
B_{i}\left(x_{1}, \ldots, x_{m}\right) & :=\frac{\left(\prod_{j=1}^{i}\left(b_{j}-x_{j}\right)\right)^{1-\alpha}}{\prod_{j=1}^{i-1}\left(b_{j}-a_{j}\right)} \int_{\prod_{j=1}^{i}\left[a_{j}, b_{j}\right]}\left(\prod_{j=1}^{i}\left(b_{j}-t_{j}\right)\right)^{\alpha-1} \\
& \cdot P_{1}\left(x_{i}, t_{i}\right) \frac{\partial f}{\partial x_{i}}\left(t_{1}, \ldots, t_{i}, x_{i+1}, \ldots, x_{m}\right) d t_{1} d t_{2} \ldots d t_{i} .
\end{aligned}
$$

Proof. By (6) we have

$$
\begin{gathered}
f\left(x_{1}, \ldots, x_{m}\right)=\left(b_{1}-x_{1}\right)^{1-\alpha}\left[\frac{1}{b_{1}-a_{1}} \int_{a_{1}}^{b_{1}}\left(b_{1}-t_{1}\right)^{\alpha-1} f\left(t_{1}, x_{2}, \ldots, x_{m}\right) d t_{1}\right. \\
-(\alpha-1) \int_{a_{1}}^{b_{1}}\left(b_{1}-t_{1}\right)^{\alpha-2} P_{1}\left(x_{1}, t_{1}\right) f\left(t_{1}, x_{2}, \ldots, x_{m}\right) d t_{1} \\
\left.+\int_{a_{1}}^{b_{1}}\left(b_{1}-t_{1}\right)^{\alpha-1} P_{1}\left(x_{1}, t_{1}\right) \frac{\partial f}{\partial x_{1}}\left(t_{1}, x_{2}, \ldots, x_{m}\right) d t_{1}\right], \quad \text { (13) }
\end{gathered}
$$


and

$$
\begin{aligned}
& f\left(t_{1}, x_{2}, \ldots, x_{m}\right)= \\
& =\left(b_{2}-x_{2}\right)^{1-\alpha}\left[\frac{1}{b_{2}-a_{2}} \int_{a_{2}}^{b_{2}}\left(b_{2}-t_{2}\right)^{\alpha-1} f\left(t_{1}, t_{2}, x_{3}, \ldots, x_{m}\right) d t_{2}\right. \\
& -(\alpha-1) \int_{a_{2}}^{b_{2}}\left(b_{2}-t_{2}\right)^{\alpha-2} P_{1}\left(x_{2}, t_{2}\right) f\left(t_{1}, t_{2}, x_{3}, \ldots, x_{m}\right) d t_{2} \\
& \left.\quad+\int_{a_{2}}^{b_{2}}\left(b_{2}-t_{2}\right)^{\alpha-1} P_{1}\left(x_{2}, t_{2}\right) \frac{\partial f}{\partial x_{2}}\left(t_{1}, t_{2}, x_{3}, \ldots, x_{m}\right) d t_{2}\right] .
\end{aligned}
$$

We plug in (14) into (13). Hence

$$
\begin{gathered}
f\left(x_{1}, \ldots, x_{m}\right)=\left(b_{1}-x_{1}\right)^{1-\alpha}\left[\frac{1}{b_{1}-a_{1}} \int_{a_{1}}^{b_{1}}\left(b_{1}-t_{1}\right)^{\alpha-1}\left(b_{2}-x_{2}\right)^{1-\alpha} .\right. \\
{\left[\frac{1}{b_{2}-a_{2}} \int_{a_{2}}^{b_{2}}\left(b_{2}-t_{2}\right)^{\alpha-1} f\left(t_{1}, t_{2}, x_{3}, \ldots, x_{m}\right) d t_{2}\right.} \\
-(\alpha-1) \int_{a_{2}}^{b_{2}}\left(b_{2}-t_{2}\right)^{\alpha-2} P_{1}\left(x_{2}, t_{2}\right) f\left(t_{1}, t_{2}, x_{3}, \ldots, x_{m}\right) d t_{2} \\
\left.+\int_{a_{2}}^{b_{2}}\left(b_{2}-t_{2}\right)^{\alpha-1} P_{1}\left(x_{2}, t_{2}\right) \frac{\partial f}{\partial x_{2}}\left(t_{1}, t_{2}, x_{3}, \ldots, x_{m}\right) d t_{2}\right] d t_{1} \\
-(\alpha-1) \int_{a_{1}}^{b_{1}}\left(b_{1}-t_{1}\right)^{\alpha-2} P_{1}\left(x_{1}, t_{1}\right) f\left(t_{1}, x_{2}, \ldots, x_{m}\right) d t_{1} \\
\left.+\int_{a_{1}}^{b_{1}}\left(b_{1}-t_{1}\right)^{\alpha-1} P_{1}\left(x_{1}, t_{1}\right) \frac{\partial f}{\partial x_{1}}\left(t_{1}, x_{2}, \ldots, x_{m}\right) d t_{1}\right] .
\end{gathered}
$$

That is we have so far

$$
\begin{gathered}
f\left(x_{1}, \ldots, x_{m}\right)=\frac{\left(b_{1}-x_{1}\right)^{1-\alpha}\left(b_{2}-x_{2}\right)^{1-\alpha}}{\left(b_{1}-a_{1}\right)\left(b_{2}-a_{2}\right)} \\
\cdot \int_{a_{1}}^{b_{1}} \int_{a_{2}}^{b_{2}}\left(b_{1}-t_{1}\right)^{\alpha-1}\left(b_{2}-t_{2}\right)^{\alpha-1} f\left(t_{1}, t_{2}, x_{3}, \ldots, x_{m}\right) d t_{1} d t_{2} \\
-\frac{(\alpha-1)\left(b_{1}-x_{1}\right)^{1-\alpha}\left(b_{2}-x_{2}\right)^{1-\alpha}}{\left(b_{1}-a_{1}\right)} \\
\cdot \int_{a_{1}}^{b_{1}} \int_{a_{2}}^{b_{2}}\left(b_{1}-t_{1}\right)^{\alpha-1}\left(b_{2}-t_{2}\right)^{\alpha-1} P_{1}\left(x_{2}, t_{2}\right) f\left(t_{1}, t_{2}, x_{3}, \ldots, x_{m}\right) d t_{1} d t_{2} \\
+\frac{\left(b_{1}-x_{1}\right)^{1-\alpha}\left(b_{2}-x_{2}\right)^{1-\alpha}}{\left(b_{1}-a_{1}\right)}
\end{gathered}
$$




$$
\begin{aligned}
& \cdot \int_{a_{1}}^{b_{1}} \int_{a_{2}}^{b_{2}}\left(b_{1}-t_{1}\right)^{\alpha-1}\left(b_{2}-t_{2}\right)^{\alpha-1} P_{1}\left(x_{2}, t_{2}\right) \frac{\partial f}{\partial x_{2}}\left(t_{1}, t_{2}, x_{3}, \ldots, x_{m}\right) d t_{1} d t_{2} \\
& -(\alpha-1)\left(b_{1}-x_{1}\right)^{1-\alpha} \int_{a_{1}}^{b_{1}}\left(b_{1}-t_{1}\right)^{\alpha-2} P_{1}\left(x_{1}, t_{1}\right) f\left(t_{1}, x_{2}, \ldots, x_{m}\right) d t_{1} \\
& \quad+\left(b_{1}-x_{1}\right)^{1-\alpha} \int_{a_{1}}^{b_{1}}\left(b_{1}-t_{1}\right)^{\alpha-1} P_{1}\left(x_{1}, t_{1}\right) \frac{\partial f}{\partial x_{1}}\left(t_{1}, x_{2}, \ldots, x_{m}\right) d t_{1} .
\end{aligned}
$$

Call

$$
\begin{aligned}
& A_{1}\left(x_{1}, \ldots, x_{m}\right):=-(\alpha-1)\left(b_{1}-x_{1}\right)^{1-\alpha} \int_{a_{1}}^{b_{1}}\left(b_{1}-t_{1}\right)^{\alpha-2} P_{1}\left(x_{1}, t_{1}\right) f\left(t_{1}, x_{2}, \ldots, x_{m}\right) d t_{1}, \\
& B_{1}\left(x_{1}, \ldots, x_{m}\right):=\left(b_{1}-x_{1}\right)^{1-\alpha} \\
& \cdot \int_{a_{1}}^{b_{1}}\left(b_{1}-t_{1}\right)^{\alpha-1} P_{1}\left(x_{1}, t_{1}\right) \frac{\partial f}{\partial x_{1}}\left(t_{1}, x_{2}, \ldots, x_{m}\right) d t_{1}, \quad(1) \\
& A_{2}\left(x_{1}, x_{2}, \ldots, x_{m}\right):=-\frac{(\alpha-1)\left(b_{1}-x_{1}\right)^{1-\alpha}\left(b_{2}-x_{2}\right)^{1-\alpha}}{\left(b_{1}-a_{1}\right)} \\
& \cdot \int_{a_{1}}^{b_{1}} \int_{a_{2}}^{b_{2}}\left(b_{1}-t_{1}\right)^{\alpha-1}\left(b_{2}-t_{2}\right)^{\alpha-1} P_{1}\left(x_{2}, t_{2}\right) f\left(t_{1}, t_{2}, x_{3}, \ldots, x_{m}\right) d t_{1} d t_{2},
\end{aligned}
$$

and

$$
\begin{aligned}
& B_{2}\left(x_{1}, x_{2}, \ldots, x_{m}\right):=\frac{\left(b_{1}-x_{1}\right)^{1-\alpha}\left(b_{2}-x_{2}\right)^{1-\alpha}}{\left(b_{1}-a_{1}\right)} \\
& \cdot \int_{a_{1}}^{b_{1}} \int_{a_{2}}^{b_{2}}\left(b_{1}-t_{1}\right)^{\alpha-1}\left(b_{2}-t_{2}\right)^{\alpha-1} P_{1}\left(x_{2}, t_{2}\right) \frac{\partial f}{\partial x_{2}}\left(t_{1}, t_{2}, x_{3}, \ldots, x_{m}\right) d t_{1} d t_{2} .
\end{aligned}
$$

We rewrite (16) as follows

$$
\begin{gathered}
f\left(x_{1}, \ldots, x_{m}\right)=\frac{\left(\left(b_{1}-x_{1}\right)\left(b_{2}-x_{2}\right)\right)^{1-\alpha}}{\left(b_{1}-a_{1}\right)\left(b_{2}-a_{2}\right)} \\
\cdot \int_{a_{1}}^{b_{1}} \int_{a_{2}}^{b_{2}}\left(\left(b_{1}-t_{1}\right)\left(b_{2}-t_{2}\right)\right)^{\alpha-1} f\left(t_{1}, t_{2}, x_{3}, \ldots, x_{m}\right) d t_{1} d t_{2} \\
+A_{2}\left(x_{1}, \ldots, x_{m}\right)+B_{2}\left(x_{1}, \ldots, x_{m}\right)+A_{1}\left(x_{1}, \ldots, x_{m}\right)+B_{1}\left(x_{1}, \ldots, x_{m}\right) .
\end{gathered}
$$


We continue with

$$
\begin{gathered}
f\left(t_{1}, t_{2}, x_{3}, \ldots, x_{m}\right) \stackrel{(6)}{=} \\
\stackrel{(6)}{=} \frac{\left(b_{3}-x_{3}\right)^{1-\alpha}}{b_{3}-a_{3}} \int_{a_{3}}^{b_{3}}\left(b_{3}-t_{3}\right)^{\alpha-1} f\left(t_{1}, t_{2}, t_{3}, x_{4}, \ldots, x_{m}\right) d t_{3} \\
-(\alpha-1)\left(b_{3}-x_{3}\right)^{1-\alpha} \int_{a_{3}}^{b_{3}}\left(b_{3}-t_{3}\right)^{\alpha-2} P_{1}\left(x_{3}, t_{3}\right) f\left(t_{1}, t_{2}, t_{3}, x_{4}, \ldots, x_{m}\right) d t_{3} \\
+\left(b_{3}-x_{3}\right)^{1-\alpha} \int_{a_{3}}^{b_{3}}\left(b_{3}-t_{3}\right)^{\alpha-1} P_{1}\left(x_{3}, t_{3}\right) \frac{\partial f}{\partial x_{3}}\left(t_{1}, t_{2}, t_{3}, x_{4}, \ldots, x_{m}\right) d t_{3} .
\end{gathered}
$$

Next plug (22) into (21). Hence it holds

$$
\begin{gathered}
f\left(x_{1}, \ldots, x_{m}\right)=\frac{\left(\left(b_{1}-x_{1}\right)\left(b_{2}-x_{2}\right)\left(b_{3}-x_{3}\right)\right)^{1-\alpha}}{\left(b_{1}-a_{1}\right)\left(b_{2}-a_{2}\right)\left(b_{3}-a_{3}\right)} \\
\cdot \int_{a_{1}}^{b_{1}} \int_{a_{2}}^{b_{2}} \int_{a_{3}}^{b_{3}}\left(\left(b_{1}-t_{1}\right)\left(b_{2}-t_{2}\right)\left(b_{3}-t_{3}\right)\right)^{\alpha-1} \\
\cdot f\left(t_{1}, t_{2}, t_{3}, x_{4}, \ldots, x_{m}\right) d t_{1} d t_{2} d t_{3}-\frac{(\alpha-1)\left(\left(b_{1}-x_{1}\right)\left(b_{2}-x_{2}\right)\left(b_{3}-x_{3}\right)\right)^{1-\alpha}}{\left(b_{1}-a_{1}\right)\left(b_{2}-a_{2}\right)} \\
\cdot \int_{a_{1}}^{b_{1}} \int_{a_{2}}^{b_{2}} \int_{a_{3}}^{b_{3}}\left(\left(b_{1}-t_{1}\right)\left(b_{2}-t_{2}\right)\right)^{\alpha-1}\left(b_{3}-t_{3}\right)^{\alpha-2} P_{1}\left(x_{3}, t_{3}\right) \\
\cdot f\left(t_{1}, t_{2}, t_{3}, x_{4}, \ldots, x_{m}\right) d t_{1} d t_{2} d t_{3}+\frac{\left(\left(b_{1}-x_{1}\right)\left(b_{2}-x_{2}\right)\left(b_{3}-x_{3}\right)\right)^{1-\alpha}}{\left(b_{1}-a_{1}\right)\left(b_{2}-a_{2}\right)} \\
\cdot \int_{a_{1}}^{b_{1}} \int_{a_{2}}^{b_{2}} \int_{a_{3}}^{b_{3}}\left(\left(b_{1}-t_{1}\right)\left(b_{2}-t_{2}\right)\left(b_{3}-t_{3}\right)\right)^{\alpha-1} P_{1}\left(x_{3}, t_{3}\right) \\
\cdot \frac{\partial f}{\partial x_{3}}\left(t_{1}, t_{2}, t_{3}, x_{4}, \ldots, x_{m}\right) d t_{1} d t_{2} d t_{3} \\
+A_{1}\left(x_{1}, \ldots, x_{m}\right)+A_{2}\left(x_{1}, \ldots, x_{m}\right)+B_{1}\left(x_{1}, \ldots, x_{m}\right)+B_{2}\left(x_{1}, \ldots, x_{m}\right) .
\end{gathered}
$$

Call

$$
\begin{array}{r}
A_{3}\left(x_{1}, \ldots, x_{m}\right):=-\frac{(\alpha-1)\left(\left(b_{1}-x_{1}\right)\left(b_{2}-x_{2}\right)\left(b_{3}-x_{3}\right)\right)^{1-\alpha}}{\left(b_{1}-a_{1}\right)\left(b_{2}-a_{2}\right)} \\
\cdot \int_{a_{1}}^{b_{1}} \int_{a_{2}}^{b_{2}} \int_{a_{3}}^{b_{3}}\left(\left(b_{1}-t_{1}\right)\left(b_{2}-t_{2}\right)\right)^{\alpha-1}\left(b_{3}-t_{3}\right)^{\alpha-2} P_{1}\left(x_{3}, t_{3}\right) \\
\cdot f\left(t_{1}, t_{2}, t_{3}, x_{4}, \ldots, x_{m}\right) d t_{1} d t_{2} d t_{3}
\end{array}
$$


and

$$
\begin{gathered}
B_{3}\left(x_{1}, \ldots, x_{m}\right):=\frac{\left(\left(b_{1}-x_{1}\right)\left(b_{2}-x_{2}\right)\left(b_{3}-x_{3}\right)\right)^{1-\alpha}}{\left(b_{1}-a_{1}\right)\left(b_{2}-a_{2}\right)} \\
\cdot \int_{a_{1}}^{b_{1}} \int_{a_{2}}^{b_{2}} \int_{a_{3}}^{b_{3}}\left(\left(b_{1}-t_{1}\right)\left(b_{2}-t_{2}\right)\left(b_{3}-t_{3}\right)\right)^{\alpha-1} P_{1}\left(x_{3}, t_{3}\right) \\
\cdot \frac{\partial f}{\partial x_{3}}\left(t_{1}, t_{2}, t_{3}, x_{4}, \ldots, x_{m}\right) d t_{1} d t_{2} d t_{3} .
\end{gathered}
$$

Thus we have proved

$$
\begin{aligned}
f\left(x_{1}, \ldots, x_{m}\right) & =\frac{\left(\prod_{i=1}^{3}\left(b_{i}-x_{i}\right)\right)^{1-\alpha}}{\prod_{i=1}^{3}\left(b_{i}-a_{i}\right)} \\
\cdot \int_{\prod_{i=1}^{3}\left[a_{i}, b_{i}\right]} & \left(\prod_{i=1}^{3}\left(b_{i}-t_{i}\right)\right)^{\alpha-1} f\left(t_{1}, t_{2}, t_{3}, x_{4}, \ldots, x_{m}\right) d t_{1} d t_{2} d t_{3} \\
& \quad+\sum_{i=1}^{3} A_{i}\left(x_{1}, \ldots, x_{m}\right)+\sum_{i=1}^{3} B_{i}\left(x_{1}, \ldots, x_{m}\right) .
\end{aligned}
$$

Working similarly we finally obtain the fractional representation formula

$$
\begin{gathered}
f\left(x_{1}, \ldots, x_{m}\right)=\frac{\left(\prod_{i=1}^{m}\left(b_{i}-x_{i}\right)\right)^{1-\alpha}}{\prod_{i=1}^{m}\left(b_{i}-a_{i}\right)} \int_{\prod_{i=1}^{m}\left[a_{i}, b_{i}\right]}\left(\prod_{i=1}^{m}\left(b_{i}-t_{i}\right)\right)^{\alpha-1} f(\vec{t}) d \vec{t} \\
+\sum_{i=1}^{m} A_{i}\left(x_{1}, \ldots, x_{m}\right)+\sum_{i=1}^{m} B_{i}\left(x_{1}, \ldots, x_{m}\right) .
\end{gathered}
$$

The proof of the theorem is now completed.

We make

Remark 8. Let $f \in C^{1}\left(\prod_{i=1}^{m}\left[a_{i}, b_{i}\right]\right), \alpha \geq 1, x_{i} \in\left[a_{i}, b_{i}\right), i=1, \ldots, m$. Denote by

$$
\|f\|_{\infty}^{\sup }:=\sup _{x \in \prod_{i=1}^{m}\left[a_{i}, b_{i}\right]}|f(x)| .
$$

We observe that

$$
\begin{gathered}
\left|B_{i}\left(x_{1}, \ldots, x_{m}\right)\right| \stackrel{(12)}{\leq} \frac{\left(\prod_{j=1}^{i}\left(b_{j}-x_{j}\right)\right)^{1-\alpha}}{\prod_{j=1}^{i-1}\left(b_{j}-a_{j}\right)} \int_{\prod_{j=1}^{i}\left[a_{j}, b_{j}\right]}\left(\prod_{j=1}^{i}\left(b_{j}-t_{j}\right)\right)^{\alpha-1} \\
\left.\cdot\left|P_{1}\left(x_{i}, t_{i}\right)\right| d t_{1} \ldots d t_{i}\right)\left\|\frac{\partial f}{\partial x_{i}}\right\|_{\infty}^{\text {sup }}
\end{gathered}
$$




$$
\begin{aligned}
& =\frac{\left(\prod_{j=1}^{i}\left(b_{j}-x_{j}\right)\right)^{1-\alpha}\left\|\frac{\partial f}{\partial x_{i}}\right\|_{\infty}^{\text {sup }}}{\prod_{j=1}^{i-1}\left(b_{j}-a_{j}\right)}\left(\prod_{j=1}^{i-1} \int_{a_{j}}^{b_{j}}\left(b_{j}-t_{j}\right)^{\alpha-1} d t_{j}\right) \\
& \cdot\left(\int_{a_{i}}^{b_{i}}\left(b_{i}-t_{i}\right)^{\alpha-1}\left|P_{1}\left(x_{i}, t_{i}\right)\right| d t_{i}\right) \\
& =\frac{\left(\prod_{j=1}^{i}\left(b_{j}-x_{j}\right)\right)^{1-\alpha}\left\|\frac{\partial f}{\partial x_{i}}\right\|_{\infty}^{\text {sup }}}{\left(b_{i}-a_{i}\right) \prod_{j=1}^{i-1}\left(b_{j}-a_{j}\right)} \frac{\left(\prod_{j=1}^{i-1}\left(b_{j}-a_{j}\right)\right)^{\alpha}}{\alpha^{i-1}} \\
& \cdot\left[\int_{a_{i}}^{x_{i}}\left(b_{i}-t_{i}\right)^{\alpha-1}\left(t_{i}-a_{i}\right) d t_{i}+\int_{x_{i}}^{b_{i}}\left(b_{i}-t_{i}\right)^{\alpha-1}\left(b_{i}-t_{i}\right) d t_{i}\right] \\
& =\frac{\left\|\frac{\partial f}{\partial x_{i}}\right\|_{\infty}^{\sup }\left(\prod_{j=1}^{i}\left(b_{j}-x_{j}\right)\right)^{1-\alpha}\left(\prod_{j=1}^{i-1}\left(b_{j}-a_{j}\right)\right)^{\alpha-1}}{\alpha^{i-1}\left(b_{i}-a_{i}\right)} \\
& \cdot\left[\int_{a_{i}}^{x_{i}}\left(b_{i}-t_{i}\right)^{\alpha-1}\left[\left(b_{i}-a_{i}\right)-\left(b_{i}-t_{i}\right)\right] d t_{i}+\frac{\left(b_{i}-x_{i}\right)^{\alpha+1}}{\alpha+1}\right] \\
& =\frac{\left\|\frac{\partial f}{\partial x_{i}}\right\|_{\infty}^{\sup }\left(\prod_{j=1}^{i}\left(b_{j}-x_{j}\right)\right)^{1-\alpha}\left(\prod_{j=1}^{i-1}\left(b_{j}-a_{j}\right)\right)^{\alpha-1}}{\alpha^{i-1}\left(b_{i}-a_{i}\right)} \\
& \cdot\left[\left(b_{i}-a_{i}\right)\left[\frac{\left(b_{i}-a_{i}\right)^{\alpha}}{\alpha}-\frac{\left(b_{i}-x_{i}\right)^{\alpha}}{\alpha}\right]-\frac{\left(b_{i}-a_{i}\right)^{\alpha+1}}{\alpha+1}+\frac{2\left(b_{i}-x_{i}\right)^{\alpha+1}}{\alpha+1}\right] \\
& =\frac{\left\|\frac{\partial f}{\partial x_{i}}\right\|_{\infty}^{\sup }\left(\prod_{j=1}^{i}\left(b_{j}-x_{j}\right)\right)^{1-\alpha}\left(\prod_{j=1}^{i-1}\left(b_{j}-a_{j}\right)\right)^{\alpha-1}}{\alpha^{i-1}\left(b_{i}-a_{i}\right)} \\
& \cdot\left[\frac{\left(b_{i}-a_{i}\right)^{\alpha+1}}{\alpha(\alpha+1)}+\frac{2\left(b_{i}-x_{i}\right)^{\alpha+1}}{\alpha+1}-\left(b_{i}-a_{i}\right) \frac{\left(b_{i}-x_{i}\right)^{\alpha}}{\alpha}\right] .
\end{aligned}
$$

We have proved for $i=1, \ldots, m$, that

$$
\left|B_{i}\left(x_{1}, \ldots, x_{m}\right)\right| \leq \frac{\left\|\frac{\partial f}{\partial x_{i}}\right\|_{\infty}^{\text {sup }}\left(\prod_{j=1}^{i}\left(b_{j}-x_{j}\right)\right)^{1-\alpha}\left(\prod_{j=1}^{i-1}\left(b_{j}-a_{j}\right)\right)^{\alpha-1}}{\alpha^{i-1}}
$$

We have established the following multivariate fractional Ostrowski type inequality. 
Theorem 9. Let $f \in C^{1}\left(\prod_{i=1}^{m}\left[a_{i}, b_{i}\right]\right), \alpha \geq 1, x_{i} \in\left[a_{i}, b_{i}\right), i=1, \ldots, m$. Then

$$
\begin{aligned}
& \mid f\left(x_{1}, \ldots, x_{m}\right) \\
& -\frac{\left(\prod_{i=1}^{m}\left(b_{i}-x_{i}\right)\right)^{1-\alpha}(\Gamma(\alpha))^{m}\left(I_{a+}^{\alpha} f\right)(b)}{\prod_{i=1}^{m}\left(b_{i}-a_{i}\right)}-\sum_{i=1}^{m} A_{i}\left(x_{1}, \ldots, x_{m}\right) \mid \\
& \quad \leq \sum_{i=1}^{m}\left\{\frac{\left\|\frac{\partial f}{\partial x_{i}}\right\|_{\infty}^{\text {sup }}\left(\prod_{j=1}^{i}\left(b_{j}-x_{j}\right)\right)^{1-\alpha}\left(\prod_{j=1}^{i-1}\left(b_{j}-a_{j}\right)\right)^{\alpha-1}}{\alpha^{i-1}}\right. \\
& \left.\cdot\left[\frac{\left(b_{i}-a_{i}\right)^{\alpha}}{\alpha(\alpha+1)}+\frac{2\left(b_{i}-x_{i}\right)^{\alpha+1}}{(\alpha+1)\left(b_{i}-a_{i}\right)}-\frac{\left(b_{i}-x_{i}\right)^{\alpha}}{\alpha}\right]\right\} .
\end{aligned}
$$

\section{REFERENCES}

[1] G. Anastassiou, M. Hooshmandasl, A. Ghasemi and F. Moftakharzadeh, Montgomery identities for fractional integrals and related fractional inequalities, J. Inequal. Pure Appl. Math., 10 (4) (2009), Article 97, 6 pages.

[2] T. Mamatov and S. Samko, Mixed fractional integration operators in mixed weighted Hölder spaces, Fract. Calc. Appl. Anal., 13 (3) (2010), 245-259.

[3] D. S. Mitrinovic, J. E. Pecaric and A. M. Fink, Inequalities for Functions and their Integrals and Derivatives, Kluwer Academic Publishers, Dordrecht, Netherlands, (1994).

[4] S. Miller and B. Ross, An Introduction to the Fractional Calculus and Fractional Differential Equations, John Wiley and Sons, USA, (1993).

(Received: October 24, 2013)

Department of Mathematical Sciences

University of Memphis

Memphis, TN 38152

U.S.A.

ganastss@memphis.edu 